\title{
Web Operating Systems
}

\author{
Ananya Tripathi \\ Amity School of Engineering and Technology, Amity University, Noida, India \\ Email: tripathi ananya90@yahoo.com \\ Received 5 April 2014; revised 10 May 2014; accepted 12 June 2014 \\ Copyright (C) 2014 by author and OALib. \\ This work is licensed under the Creative Commons Attribution International License (CC BY). \\ http://creativecommons.org/licenses/by/4.0/

(c) (i) Open Access

\begin{abstract}
One of the upcoming topics that have rapidly gained popularity these days in the area of operating system is a Web or Online Operating System. The implementation of this technology is based on the Internet and distributed computing. The objective of this technology is to deliver the full benefit of the World Wide Web. Online Operating Systems include mechanisms for persistent storage, remote process execution, client management, authentication and security. This paper presents an overview of a typical Online OS. It describes its services provided, the underlying technologies exploited, advantages of using it and some of its practical applications. Additionally, the paper discusses some of the issues and concerns surrounding the implementation and design of an Online OS.
\end{abstract}

\section{Keywords}

Web OS, Online OS, Distributed Computing Systems

\section{Subject Areas: Distributed Computing}

\section{Introduction}

Development of operating systems enabling global computing is an upcoming issue these days. Such an operating system is called the Web Operating System or Online Operating System. These terms have been used interchangeably in this paper.

To start off with, an operating system (OS) is a special kind of program that organizes and controls computer hardware and software. It acts as an interface between the user and the machine. They interact directly with computer hardware and serve as a platform for other applications.

A Web Operating System or an Online Operating System on the other hand is a user interface that allows users to access a desktop and applications installed on the Web. It maybe considered being similar to the user interface of traditional computer operating systems like Windows or Linux but it does not directly interact with the 
computer's hardware resources. The user must still have a traditional OS on his or her computer [1]. It provides basic services such as a GUI, a virtual file system, access control management and possibilities to develop and deploy applications online. This in turn finishes the need for high configuration hardware, thus cutting down on the cost.

Some of the major reasons for the Online Operating System gaining rapid popularity among its users are its reliability, availability, fault tolerance, load sharing, and function sharing. Among its developers the fact that it employs simple technologies like Flash and AJAX, is making it an upcoming issue these days [2].

At present there are dozens of Web operating systems to choose from. Some of them offer a wide range of services, while others are still in development and only provide limited functionality. In some cases, there may be a single ambitious programmer behind the project. Other Web operating systems are the product of a large team effort. Some are free to download, and others charge a fee. Web operating systems can come in all shapes and sizes. For instance Cloudo, EyeOS and G.ho.st (Global Hosted Operating SysTem) are the top three Web based operating systems [3].

Some of the immediate advantages of using an Online OS are less requirement of hardware as the physical space for storing data at frontend will no longer be required; portability of data; facility of exploiting a variety of applications altogether. Also in the long run, keeping in mind these immediate advantages the working cost would ultimately come down.

This paper primarily focuses on the basic functioning, services provided by an Online OS, the technologies put together to build it, the advantages provided to the users and some issues regarding its implementation.

The paper has been organized into 7 sections as follows. Section 2 gives an overview of the services provided by a typical Online OS. The overview includes a general description of what an Online OS does and the services it provides to its users. Section 3 addresses the underlying technologies used to implement and design an Online OS. Section 4 deals with the concerns of using an Online OS and finally. Section 5 makes a note of the various advantages of using a Web OS and discusses a few of its applications. Section 6 and 7 give away the conclusion and references respectively.

\section{Services}

Web operating systems can be considered as implementations of the concept of cloud computing. They serve as interfaces to distributed computing systems, particularly cloud computing systems. They have the plus point of being absolutely mobile in nature. Instead of being fixed to a single location i.e. the client workstation, services can dynamically be provided through the Internet computing resources and all the way to the client [4]. The ultimate goal is to provide a portable platform that allows the user to benefit from the computational potential offered by and on the web.

\subsection{Process}

In these systems, the client is provided computer services through an Internet connection. The provider runs a set of interconnected systems that include application servers and databases.

Figure 1 depicts a set of interconnected computers demonstrating the concept of distributed computing.

Web browsers like Firefox or Internet Explorer may be used for accessing some Online OS whereas some of them may require a separate program to be downloaded, which creates a system-specific client. In either case, users access programs that are stored not on their own computers, but on the Web. After accessing them, a user account that is analogous to an e-mail account has to be created which reserves some amount of space in the cloud or Internet for the user for a particular user session. This account serves as the user's own space in the enormous cloud and allows him or her to exploit the various applications made available by the particular Online OS. When the user chooses to run a certain application, the computer sends a request to the system's control node- a special server that acts as a system administrator. The control node interprets the request and connects the user's client to the appropriate application server or database.

Also, each server command is sent as javascript as the user interacts with the OS. As actions (such as launching an application) are performed using AJAX, the OS sends event information to the server. The server then sends back tasks for the client to do in XML format, such as drawing a widget. On the server, most Web OSs uses XML files to store information. This makes it simple for a user to set up on the server, as it requires zero configurations other than the account information for the first user, making it simple to deploy. To avoid any 
Distributed computing systems

commonly spread the workload over several computers. Each computer's own processor would then process its own share of work independently.

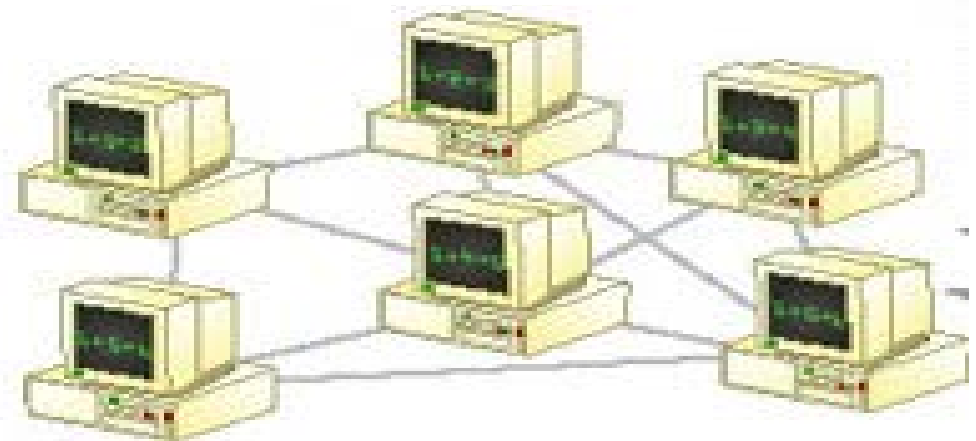

Figure 1. Example of a typical distributed computing system.

collision of the information, each user's information and settings are stored in different files [5].

\subsection{Service Components}

Practically any program that could be run on a computer's desktop can be made available to the user on the Web operating systems. Some of the most common applications include:

- E-mail.

- File management.

- Games.

- Instant messaging programs.

- Photo, video and audio editing.

- Spreadsheet programs.

- Word processing programs.

They are made available to the user as soon as they create an account with the OS [6].

With traditional computer operating systems, users are required to install applications to be able to use them. These applications that exist on the computer's hard disk drive run by accessing the processing power of the computer's central processing unit (CPU) and by sending electronic requests to the OS of the computer.

\section{Underlying Technologies}

The user side of Web OS software, whether run through a Web browser or a system-specific client, runs on top of the computer's OS. While programming the software developers must keep in mind the fact that this system ultimately has to act like a desktop OS and so its look and feel must be retained. Normally a Web OS might look a lot like a traditional OS, but it does not manage the computer's hardware or software.

While the goal of a Web OS is to provide an experience similar to using a desktop OS, there are no hard and fast rules for how to make that happen. The two most popular approaches employ Flash technologies or Asynchronous JavaScript and XML (AJAX) technologies.

\section{Building an Online Operating System}

Flash provides a set of technologies that enable programmers to create interactive Web pages. It is a technology that uses vector graphics. Vector graphics record image data as a collection of shapes and lines rather than individual pixels, which allows computers to load Flash images and animation faster than pixel-based graphics [7]. The end user accessing the flash content does not have to wait for the entire file to download on to the computer before accessing it as the Flash files stream over the Internet. The fact that more than 98 percent of all computers connected to the Internet have a Flash player installed [8]. This makes Flash an attractive approach for employing it to develop the concept by many programmers. They can create a Web OS knowing that the vast majority of computer users will be able to access it without having to download additional software.

AJAX technologies rely on hypertext markup language (HTML), the JavaScript programming language, 
Cascading Style Sheets (CSS) and eXtensible Markup Language (XML). It's a browser technology. The HTML language is a collection of markup tags programmers use on text files that tell Web browsers how to display the text file as a Web page. CSS is a tool that gives programmers more options when tweaking a Web site's appearance. Programmers can create a style sheet with certain attributes such as font style and color, and then apply those styles across several Web pages at once. JavaScript is a programming language that allows applications to send information back and forth between servers and browsers. XML is a markup language, which means programmers use it to describe the structure of information within a file and how it relates to other information [9].

The "asynchronous" aspect of AJAX means that AJAX applications transfer data between servers and browsers in small bits of information as needed. The alternative is to send an entire Web page to the browser every time something changes, which would significantly slow down the user's experience. With sufficient skill and knowledge, a programmer can create an AJAX application with the same functions as a desktop application.

The information intended to be safe by the user is stored on the databases connected to that particular OS or in broader sense, the Internet. Also, some Online Operating Systems provide the option to save information on to the local hard disk drive.

\section{Concerns and Issues}

\subsection{Implementation Issues}

Handling concurrent users is done by implementing the concept of distributed systems. As already mentioned, the Online OS serve as the interfaces for distributed computing systems. A distributed system consists of multiple autonomous computers that communicate through a computer network. The computers interact with each other in order to achieve a common goal [10]. Distributed computing also refers to the use of distributed systems to solve computational problems. In distributed computing, a problem is divided into many tasks, each of which is solved by one computer [11]. Employing this model for implementing the Online OS have the simple issues of reduced cost, increased reliability and high performance behind it. The use of a distributed system is beneficial for practical reasons. For example, it may be more cost-efficient to obtain the desired level of performance by using a cluster of several low-end computers, in comparison with a single high-end computer. A distributed system can be more reliable than a non-distributed system, as there is no single point of failure [12]. Also, they serve as the best option to handle the continuous requests of the concurrent users worldwide.

\subsection{Concerns Related to Security}

A common concern about Online Operating Systems is that they require a third party to be trusted by the user for storing their personal and confidential data. On the contrary, the developers are making their best efforts to resolve this concern, as it is also a question of their repute and prestige. Also, the use of distributed systems makes the concept of an Online OS even more secure.

\section{Advantages and Applications}

This section covers the various advantages of using an Online OS and some of its enterprise applications.

\subsection{Advantages}

The user benefits in the following ways:

1) Mobility: An implementation of the anytime, anywhere idea. The user can simply log on to the OS using an Internet connection, thus minimizing the requirements to just a computer and a connection to the Web.

2) Software management: It is the responsibility of the provider to buy and update all software from time to time thus ensuring that the users are always working on their current versions.

3) High availability: Minimizes the need for latest hardware requirements and updating the system from time to time. Since it is a completely web based solution, the client is free to log on to another system and resume working in case of failure of the current working system.

\subsection{Applications}

Some of the practical applications of the concept of a Web operating system are described in this section. 
1) An organisation may develop a new Web OS of its own and make it functional on its local intranet, thus making the working platform of its employees uniform.

2) This also reduces the cost of buying the licensed versions of the various softwares for each employee group, and also updating them regularly.

3) Web operating systems can also simplify collaborative projects. Many Web operating systems allow users to share files. Each user can work from the file saved to the system's native network. This is an attractive alternative to organizing multiple copies of the same file and then incorporating everyone's changes into a new version.

\section{Conclusions}

Online Operating Systems have the potential of being an important future aspect of the distributed computing system for the Internet. It also offers high reliability, security and manageability.

These operating systems do not replace the computer's native OS. They completely depend on traditional computer operating systems to work. An Online OS allows the user to access applications stored not on the computer, but on the Web. Since these operating systems are not tied to any specific computer system or device, Web applications and data can be accessed from any device connected to the Internet. This clearly implies that this concept of an Online Operating System will run as long as the Internet exists. This also means that the user can access the Web OS on one computer, create a document, save the work and then access it again later using a completely different machine. Thus these operating systems offer users the benefit of mobile accessibility of data since it is not tied down to a specific computer.

\section{References}

[1] Kropf, P.G. (1999) Overview of the Web Operating System (WOS) Project. 1999 Advanced Simulation Technologies Conference (ASTC 1999). San Diego, 350-356.

[2] Adobe (2007) Flash Player Penetration. http://www.adobe.com/products/player_census/flashplayer/

[3] (2011) The Top 10 Web Based OS Page. http://techie-buzz.com/internet-tools/top10-web-os-you-can-load-on-the-browser.html

[4] Krone, O. and Schubiger, S. (1999) WebRes: Towards a Web Operating System. Kommunikation in Verteilten Systems, 418-429.

[5] (2011) The How Stuff Works Website. http://www.howstuffworks.com/

[6] (2011) What Is a WebOS? http://www.zdnet.com/blog/web2explorer/what-is-a-webos/178

[7] CNET News (2007) Is the "Web OS” Just a Geek's Dream? Is-the-Web-OS-just-a-geeks-dream/2100-7345_3-6174111.html

[8] Adobe. Flash Player Statistics. http://www.adobe.com/products/player_census/flashplayer/

[9] What Is AJAX? http://webdesign.about.com/od/ajax/a/aa101705.htm

[10] Ghosh, S. (2007) Distributed Systems: An Algorithmic Approach. Chapman \& Hall, CRC, Boca Raton.

[11] Godfrey, B. (2002) A Primer on Distributed Computing. http://www.bacchae.co.uk/docs/dist.html

[12] Elmasri, R. and Navathe, S.B. (2000) Fundamentals of Database Systems. 3rd Edition, Addison-Wesley, Boston. 